\title{
PLEIADES HR IN FLIGHT GEOMETRICAL CALIBRATION : LOCATION AND MAPPING OF THE FOCAL PLANE
}

\author{
Françoise de LUSSY ${ }^{\mathrm{a}}$, Daniel GRESLOU ${ }^{\mathrm{a}}$, Cécile DECHOZ ${ }^{\mathrm{a}}$, Virginie.AMBERG ${ }^{\mathrm{a}}$, Jean Marc DELVIT ${ }^{\mathrm{a}}$ Laurent LEBEGUE $^{\mathrm{a}}$, \\ Gwendoline BLANCHET ${ }^{\mathrm{a}}$, Sebastien FOUREST ${ }^{\mathrm{a}}$, \\ ${ }^{a}$ CNES, Centre spatial de Toulouse ,18 Avenue Edouard Belin, 31401 Toulouse Cedex 4 (françoise.delussy@cnes.fr) \\ Commission I, Special Session: ISPRS and IAA \\ Pléiades System and Acquisition Capability
}

KEY WORDS: Image Quality, Geometry, Location, Calibration, Pleiades-HR

\begin{abstract}
:
The Pleiades system, ORFEO system optical component (Optical and Radar Federated Earth Observation) consists of a constellation of two satellites for very High Resolution panchromatic and multispectral optical observation of the Earth. Its mission is to cover all European civilian needs (mapping, tracking floods and fires) and defence in the category of metric resolution: $0.7 \mathrm{~m}$ Nadir. The first Pleiades satellite was launched at the end of last year.

One of the key objectives of the Pleiades HR (PHR) project is to achieve a location accuracy that will allow the use of images in GIS (Geographical Information System) without geometrical model improvement by refining on ground control points. The image location without refined model was specified with the precision of the most commonly used tool ie the civil GPS. So the location accuracy has been specified at less than $12 \mathrm{~m}$ for $90 \%$ of the images on a nominal satellite configuration.

Very special care has been taken all along the PHR project realization to achieve this very good location accuracy. The final touch is given during the in-orbit commissioning phase which lasts until June 2012.

The geometric quality implies to tune the parameters involved in the geolocation model (geometric calibration): besides attitude and orbit restitution tuning (not considered here), it consists in estimating the biases between the instrument orientation and the AOCS reference frame, and also the sight line of each detector in the focal plane. This is called static geometrical model. The analysis of dynamic perturbations outside of the model are the second most important image quality objective of in-flight commissioning, not described in this paper.

Finally "image quality assessment" consists in evaluating the image quality obtained in the final products. For geolocation model, it is quantified by the absolute geolocation and the pointing accuracies, and it is a main contributor in length alteration and planimetric and altimetric accuracies.

In this paper we will present both the different practices we have adopted (their advantages, limitations and complementarities) and the means we are using for the operational assessment of the location quality of PHR images. We will focus on the innovative methods and mention the improvements in progress. To conclude, we will present the very first accuracy results assessed after PHR1A launch on L1 and Sensor products.
\end{abstract}

\section{INTRODUCTION}

PLEIADES-HR is the highest resolution civilian Earth observing system ever developed in Europe. This imagery program is conducted by the French National Space Agency, CNES. It is the French part of the French-Italian ORFEO program which also comprises COSMO-SkyMed, an Italian high-resolution radar system. It will operate two agile satellites designed to provide optical images to civilian and defence users. The first Pleiades satellite was successfully launched December 17, 2011. Images are simultaneously acquired in Panchromatic (PA) and multi-spectral (XS) mode, which allows, in nadir acquisition condition, to deliver $20 \mathrm{~km}$ wide, false or natural coloured scenes with a $70 \mathrm{~cm}$ ground sampling distance after PA+XS fusion. Coverage will be almost worldwide with a revisit interval of $24 \mathrm{~h}$ for 2 satellites.

Since Pleiades 1A launch we are pleased to admire the images it provides and to work carefully to tune finely their processing. It's now time to make a status on one of its most important characteristics: the accuracy of the location given by its models.
The in-orbit commissioning phase is not yet completed as it must be carried out until end of June. But the results already obtained are satisfactory and areas for improvement are being put in place. Therefore valid and rather complete results can be usefully presented about location performances observed during these first months of life and also about future perspectives. This presentation therefore focuses on Pleiades products location through its physical model.

After a quick presentation of Pleiades specifications we will rapidly describe the satellite elements that participate to Pleiades location, and then highlight the particularities of the ground system implemented to assure these performances. Eventually we will finely detail the particularities of Pleiades location tool: taking into account the aberration of light on Earth sites and the average atmospheric refraction.

We will then focus on the method put in place to calibrate the alignment bias between camera orientation and the line of sight returned by Pleiades attitude, and on the system performance measurement. 
And then we will show the present performance in term of a priori and a posteriori location.

\section{PHR ABSOLUTE LOCATION ACCURACY REQUIREMENTS}

Absolute location represents the position of the scene on Earth surface. This criterion can be split into two criteria: a priori location and a posteriori location.

A priori location involves the ability to acquire a given site. This requirement is associated with the programming function and concerns the ability of the system to optimally point a target on ground.

The theoretical location accuracy stands for the accuracy required for pointing the central pixel of each image row of each spectral band, throughout the duration of an image acquisition segment. In other words, it must be possible to image any point on ground that is accessible from an orbit with the required accuracy.

The specification concerns this error after calibration of the inflight bias. This specification is split into a centring specification $\mathbf{5 0 0}$ meters at $\mathbf{9 9 . 7 \%}$ positioning along the swath, thus perpendicular to the scanning direction), and a specification for positioning the image along the scanning direction lower than $\mathbf{1 0 0 0}$ metres at $\mathbf{9 9 . 7 \%}$.

This criterion makes demands on orbital positioning, swath, time-stamping and pointing.

The main contributor to this criterion is orbital positioning. But a new need has arisen for the programming of stars: a need to accurate pointing attitude and guidance.

A posteriori location involves the knowledge location of a scene acquired in a terrestrial reference frame. For Pléiades system, the main specification is to avoid the need of refining the models by using ground control points GCP or reference image. So the a posteriori location error stands for the deviation between the real position of any point on an image and its estimated position using the geometrical model of the image. The accuracy will depend on both the accuracy of the reference DTM and the view angle.

The specification concerns the value of this error after in-flight bias geometric calibration. The positions are expressed with respect to the reference ellipsoid and the altitude value is given by a perfect DTM. The a posteriori location error must be less than 12 meters as probable value $(90 \%$ of the images) and better than $\mathbf{2 4}$ meters for $\mathbf{9 9 . 7 \%}$ of the images, performed with the viewing line depointed within a maximum half-angle cone of $30^{\circ}$ around the nadir viewing direction.

This criterion makes demands on data provided by the system e.g. orbit, time-stamping, attitude, lines of sight (instrument instability, thermo-elastic effect), accuracy of adjustment of misalignment between line of sight and attitude frame.

All the geometric image quality specifications are formulated as probable values, statistical values corresponding to a $90 \%$ probability that they will not be exceeded or max to $99.7 \%$. This evaluation is obtained after processing a large number of image points throughout the satellite life. Geometric criterion budget and performance are estimated statistically by Monte Carlo drawing and simulation on scenarios of use and on a large number of image segments until distribution convergence to $90 \%$ and $99.7 \%$.

\section{CHARACTERISTICS OF THE PLEIADES SATELLITE AND SYSTEM RELATED TO LOCATION}

\subsection{Satellite, System and image characteristics}

\section{Orbit :}

Orbit determination and time stamping are computed onboard with centimeter accuracy by the two redundant Doris navigators, taking into account the attitude of the satellite (provided by the AOCS board) in order to correct their distance measurements.

The expected accuracy is better than $2 \mathrm{~m}$, drift lower than $1 \mathrm{~cm}$ per second.

Extrapolation of the orbit by the ground system for preparing the programming is corrected by automatic rectification of the segment time, function of the delay or advance of the previous ascending node. This mechanism limits the main item of the a priori location budget. Since launch, it has not been used after the two first maneuvers.

\section{Attitude :}

Attitude control is performed by means of a cluster of 4 control moment gyroscopes which sequence the images acquisition far more quickly than with reaction wheels.

Users require a high level of system location accuracy preferably without ground control points. This location performance needs an extremely accurate determination of the imaging geometric model

The attitude control system uses 4 fiber-optic gyroscopes and 3 star trackers SED36 to provide restitution accuracy compatible with the system location requirements.

In addition, the system has some means to improve attitude and misalignment between line of sight and attitude frame:

- A ground centre receives AOCS measurements (star trackers and gyro) and can improve or test new solutions for attitudes determination. In delayed time using measurements before and after, these attitudes could then be released into the system.

- Image parameter files (GIPP) allow the system to adjust the alignment bias and even to track changes to follow a seasonal or temporal evolution. An update is underway to allow a further improvement according to PSO correction to account for thermo elastic phenomena.

The physical location model takes into account the physical phenomena that affect more than $1 \mathrm{~m}$ like atmospheric refraction and light aberration between the satellite and the Earth.

\subsubsection{Atmospheric refraction :}

When a light beam passes through the atmosphere layers with different refractive indexes due to the variation in air density as a function of altitude, it is deflected in accordance with the law of Descartes:

$$
n_{1} \sin \alpha_{1}=n_{2} \sin \alpha_{2}
$$

wherein $\mathrm{n} 1$ and $\mathrm{n} 2=$ indices of the two sides of the transition, $\alpha_{1}$ et $\alpha_{2}=$ angles of incidence and emergence of the beam. 


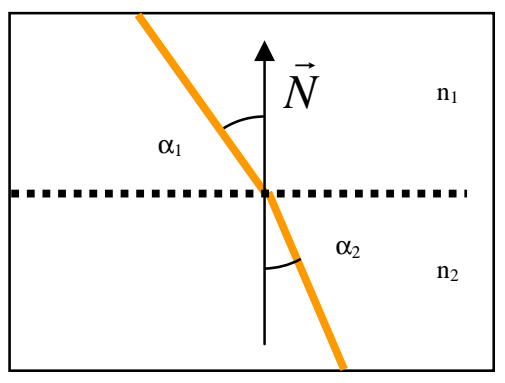

Figure 1. Atmospheric refraction between two layers

Typically, the ground deviation of a light ray whose incidence angle at the top of the atmosphere is $30^{\circ}$, is 1.9 meters , and grows up to 5 meters for an incidence angle of $45^{\circ}$, due to the atmospheric refraction. The light wavelength is a significant factor for small values (in blue and below), and much less from the red and beyond. The correction of this deviation along with a medium ground pressure allows to correct this error to first order. Under conventional conditions of imaging, the remaining error due to the lack of knowledge of the local pressure (on the beam deflection) is of the order of several centimetres, and thus negligible.

The correction is not integrated into the physical model, it is realized in localization software using the physical model. And it is taken into account in the rational model of perfect sensor. Data to perform this average correction are supplied in a ground image parameters file with the products, in order to allow the user to better correct with the local atmospheric data at the time of its own image.

\subsubsection{Light aberration:}

At the instant of any observation of an object, the apparent position of the object( i.e the direction in which it is seen by an observer on the moving observer frame) is displaced from its true position (i.e. the direction of the straight line between observer and observed at the instant of observation). This displacement depends upon the transverse component of the velocity of the observer with respect to the vector of the incoming beam of light: the difference between those two positions is caused mostly by aberration.

This phenomenon is classically taken into account in star tracker processing, which delivers absolute attitude information from stellar observation corrected of this aberration (approximately varying between -20 arcseconds and +20 arcseconds or about $100 \mu \mathrm{rad})$.

For Earth observation by satellite, the motion to take into account is the satellite speed in an Earth "inertial" geocentric reference frame (Vsat around $7 \mathrm{~km} / \mathrm{s}$ ) and the motion of the observed object due to the Earth rotation (Vobject between 0 and $500 \mathrm{~m} / \mathrm{s}$ ) in the same reference. The motion corresponding to camera movement in orbital frame with specific guidance can be neglected: $<1 \mathrm{~m} / \mathrm{s}$.

As shown on the DR1 reference, the Lorentz transformation with its relativist form can be simplified because of relative velocity amount in front of the light speed.
Magnitude and direction deviation can be easily calculated by the following expression :

$$
\vec{w}=\left(\vec{u}-\frac{\overrightarrow{V r}}{c}\right)
$$

And the relative velocity can be computed with:

$$
\vec{V} r=\left[\vec{V}_{i} s a t\right]_{I T R F}-\left[\left(\frac{d(\overrightarrow{O Q})}{d t}\right)_{R i}\right]_{I T R F}=\left[\vec{V}_{i} s a t\right]_{I T R F}-\Omega_{T}\left[\begin{array}{l}
-y_{q}-v z_{q} \\
x_{q}-u z_{q} \\
v x_{q}+u y_{q}
\end{array}\right]
$$

Where $\mathrm{Q}$ is the terrestrial object and $\mathrm{O}$ is the Earth center, $\mathrm{u}$ and $\mathrm{v}$ are the polar orientation parameters (IERS) and Vi sat is the inertial satellite velocity in an Earth-centered inertial frame.

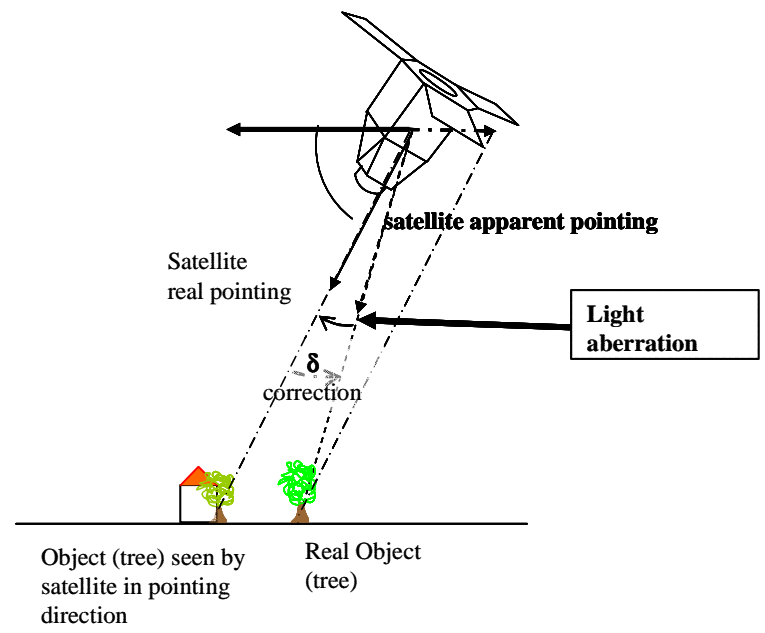

Figure 2: light aberration between satellite and ground

The deviation depends on the viewing orientation $\vec{U}$ with roll $\left(\psi_{x}\right)$ or pitch $\left(\psi_{y}\right)$.

Fig.3 shows the deviation magnitude versus roll and pitch angles. The maximum deviation is on Nadir acquisition and is around $25 \mu \mathrm{rad}$.

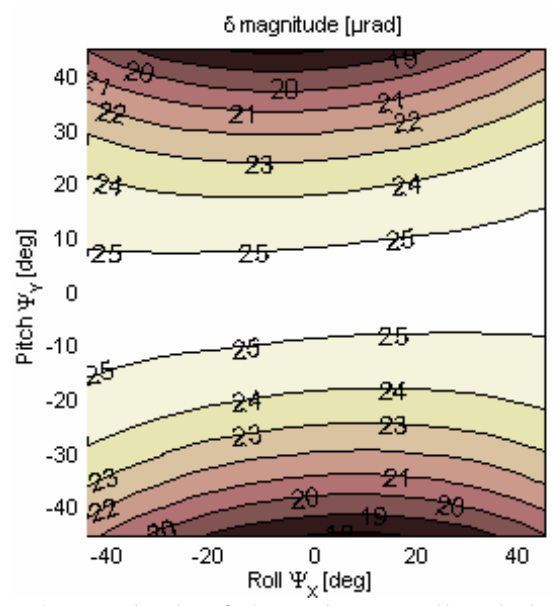

Figure 3 Magnitude of aberration vs roll and pitch angles 
As we can notice the main direction of the aberration correction is the satellite velocity.

On SPOT family satellites, the Earth aberration are not corrected but the phenomenon is calibrated by a mean value during commissioning in the corrective rotation (bias) on the camera orientation.

The Pleiades satellite agility allows different orientations of image acquisition like North-South, East-West or West-East and so on. A correction by a mean bias rotation in the camera frame is erroneous as we can see in the figures below.

On the left side of Fig.4 is depicted the off roll and pitch instrumental axis (satellite axis) orientation for three ground image orientation: Nadir, East-West and West-East and the satellite velocity and then direction of light aberration correction.

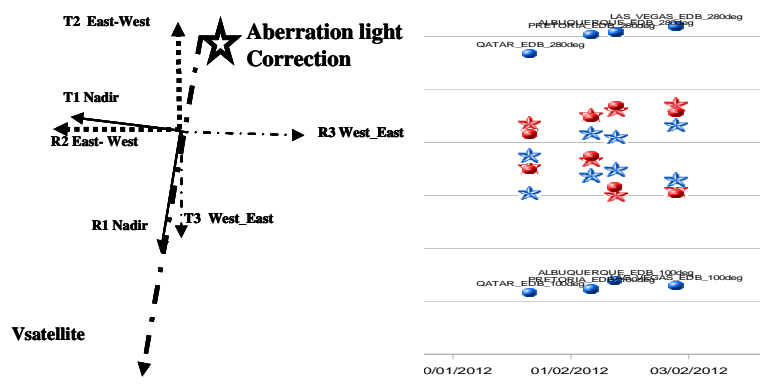

Figure 4. Light aberration correction and various images orientation (instrument yaw)

On the right side of Fig.4 is shown the bias in roll (blue) and pitch (red) for some image couples at the same location but with inverse orientation East-West and West-East. The biases without light aberration correction (circle) have a great difference in roll axis about $45 \mu \mathrm{rad}$ in the couple. The biases with light aberration correction (stars) are consistent.

So for Pleiades system a quaternion for aberration correction is evaluated for each attitude quaternion and then the attitude quaternion is corrected and given in the physical model of the raw sensor product.

\section{THE IN-FLIGHT COMMISSIONING WITH GCP} SITES

The assessment of the image quality and the calibration operation is performed by CNES Image Quality team during commissioning. Geometric activities deal with the geometric model calibration, assessment of localization accuracy, focal plane cartography, multi-spectral and multi-temporal overlapping, static and dynamic stability, planimetric, and altimetric accuracy.

Most of these results will also contribute to the tuning of the ground processing parameters in order to fit the images to the users needs.

Then we focus on the tuning of the location model parameters.

\section{Orientation biases of line sight calibration}

For each detector in the focal plane a line-of-sight model defines its orientation with respect to the sensor, i.e. the two angles which can be used to reconstruct its line of sight (a straight line linking the centre of the detector to the instrument's optical axis). These lines of sight are supposed to be stable over time, with the exception of the ground-flight transition stage (launch vibrations, change to $0 \mathrm{~g}$, outgassing from structures, etc.), and are therefore calibrated on the ground (to check that they comply with specifications and to define initial values) and then in flight.

Estimating the biases between the instrument orientation and the frame estimated by AOCS, (and also sight line of each detector in this instrument frame) is the main geometric model improvement during in-orbit commissioning .

Three methods are used for Pleiades satellite:

- The nominal and classic methods to improve the model using external information with ground control points or landmarks, whose terrain and image coordinates are well known. They enable absolute adjustment of the image to match the terrain. This method needs an accurate and large GCP database and the pointing input on the pixels images (with correlation help).

- An innovative method called "auto-reverse" uses a couple of image from the same orbit with ground orientation quasi inverse. This method enables to asses Roll and pitch biases without external information (with a classic DTM).

- A new method uses stars like automatic GCP.

[4] and [5] describe these last two methods . They are very attractive and will be very useful but are not my purpose in this paper.

The nominal method consists of refining for each segment model with roll and pitch misalignment biases and time drift on the same axis as unknown parameters.

To improve the observability of yaw bias and the focal length correction we used a multi-pass coverage of $200 \mathrm{~km}$ wide (10 PHR segments). This block of spatio-triangulation is equipped with ground control point at each end and some of them also in the middle, and with tie points in the overlap areas.

Observing theses biases closely on a lot of different sites and shoots allows to improve calibration accuracy and to highlight temporal and seasonal and also orbital variations in order to incorporate them into the model.

CNES uses a large and accurate GPS GCP database, covering a wide range of latitudes for theses orbital and seasonal observations and longitudes to allow to acquire a few sites daily.

So we have a little over 20 sites with a sufficient number of ground control points or landmarks (about twenty) to allow to refine the image model by spatio-triangulation. These landmarks have various but well known altimetric and planimetric accuracy, generally about or better than one meter.

The a posteriori analysis of median misalignments and residuals after spatio-triangulation process and a lot of acquisitions allow to do a close monitoring of the observed precision of these landmarks.

In addition to these sites for misalignment tuning, we have several sites with very little ground control points but very precise, these sites are only used for performance computation.

We had planned two steps of needs in terms of programming, production and planning . :

- During In-Flight Commissioning (RV) (2 months) about 50 segments equipped by month 
- After from 2 months to 6 months 25 to 35 segments equipped by month

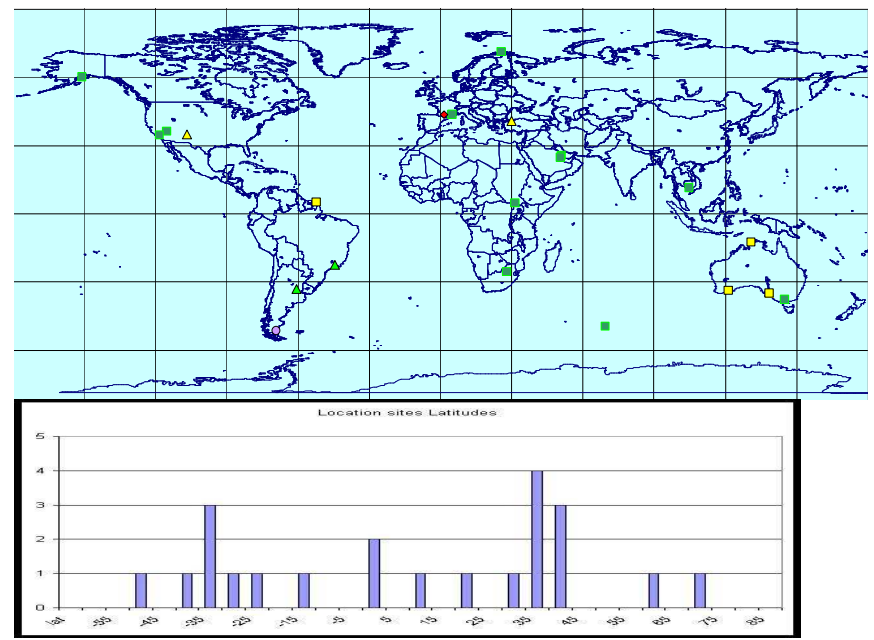

Figure 5 : Location sites position

In fact the importance of location accuracy requirements, the hydric desorption behavior of the instrument in its beginning of life led us to increase sharply by a factor of about 2 theses acquisitions and not to reduce our efforts until the performances are stabilized and improved.

\section{LOCALIZATION PERFORMANCE}

The first steps of adjusting inter direction biases of star trackers and gyroscopic scale factors have rather rapidly brought very good beginning of life performance, before calibration of instrumental biases. A first correction of theses biases have been carried out successfully on board as shown below. Fig $\mathrm{xx}$ represents the average roll and pitch shifts in $\mu$ rad for each image.

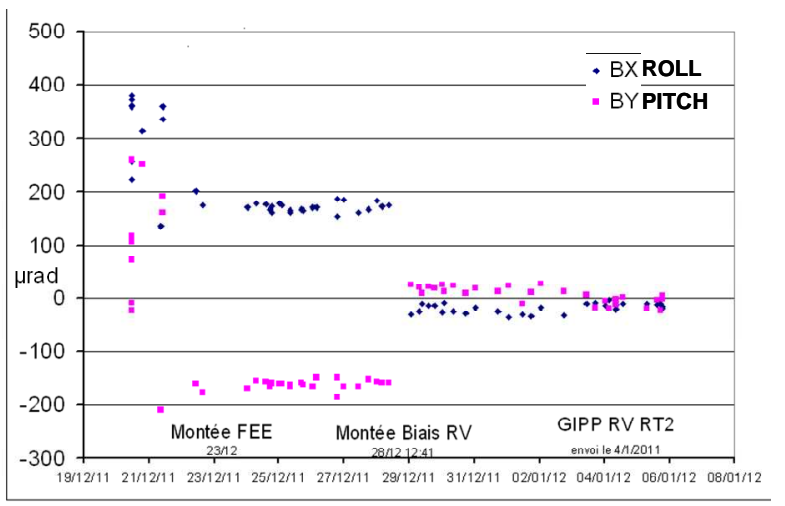

Figure 6 Misalignments improvement at the beginning of life

The pointing performance were measured by comparing the request up to the satellite and the location given by the model shooting. This performance is much better than the specification because of the very good prediction and measurement of the orbit: $80 \mathrm{~m}$ to $90 \%$ since the $311 \mathrm{PdV}$ on $05 / 01 / 2012$. And even with the beginning of life since 20/12 (428PdV), they are of 128 meters at $90 \%$
Biases tracking showed a temporal evolution stronger than expected for the water desorption, and a dispersion due to sensitivity to AOCS thermoelastic effects.

This transient behavior is thoroughly monitored in order to improve the a posteriori localization performance. Between the launch and May 25, 2012, the localization assessment is better than $16.5 \mathrm{~m}$ for $90 \%$ of images and 10m RMS.

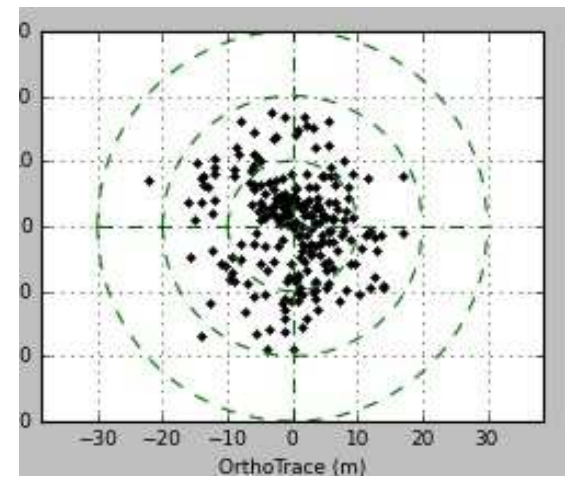

Figure 7 : A posteriori localization performance assessed April 5,2012

Because of AOCS calibration still in progress, linear drifts in the image were $1 \mu \mathrm{rad}$ per second on roll direction and $0.8 \mu \mathrm{rad}$ per second on pitch direction in the same period .

Performance will be updated in the coming months after complete characterization of the phenomena. But an already checked Improvement will achieve a better assessment of localization than 12 meters to $90 \%$, performed with the viewing line depointed less than $30^{\circ}$. It will be available for month of October.

\section{REFERENCES}

[1] L.Lebègue \& al, "Pleiades-HR Image Quality commissioning foreseen methods", IGARSS Honolulu 2010.

[2] P. Kubik \& al, "First in-flight results of Pleiades 1A innovative methods for optical calibration", ICSO Ajaccio 2012.

[3] S. Fourest \& al, "Star-based methods for Pleiades-HR commissioning”, ISPRS Melbourne 2012.

[4] D. Greslou \& al, "Pleiades-HR innovative techniques for Geometric Image Quality Commissioning", ISPRS Melbourne 2012.

[5] JM. Delvit \& al, "Attitude assessment using Pléiades-HR capabilities”, ISPRS Melbourne 2012.

[6] A. Gleyzes \& al, "Pleiades system architecture and main performances", ISPRS Melbourne 2012.

[7] D. Greslou \& al, "Ligth aberration effect in HR geometric model", ISPRS Beijing 2008

[8] F. de Lussy \& al, "Pleiades -HR Images System products and geometric accuracy”, ISPRS Hannover2005. 